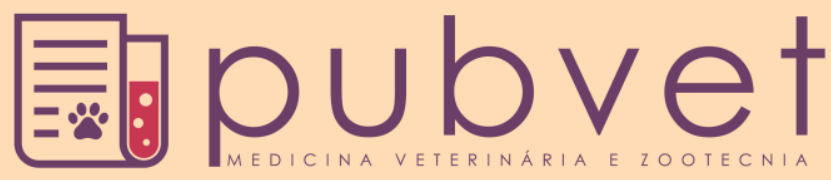

https://doi.org/10.22256/pubvet.v12n3a45.1-5

\title{
Diagnóstico por tomografia computadorizada da extrusão de disco intervertebral em paciente geriatra: Relato de caso
}

\author{
Jessyka Andréa Nascimento de Carvalho Almeida ${ }^{1 *}$, Tiago Tavares Brito de Medeiros ${ }^{\bullet 2}$, \\ Artur da Nóbrega Carreiro ${ }^{\circledR}$, Edson Mauro da Cunha ${ }^{4}$, Débora Vitória Fernandes de \\ Araújo ${ }^{\bullet}$, Brunna Muniz Rodrigues Falcão ${ }^{\bullet}$, Ana Yasha Ferreira de La Salles ${ }^{\bullet}$, \\ Danilo José Ayres de Menezes ${ }^{0}$ 6-7 \\ ${ }^{I}$ Aluna de Pós-Graduação de Clínica Médica e Cirúrgica de Pequenos Animais, Equalis, Natal, Rio Grande do Norte, Brasil. \\ ${ }^{2}$ Mestre em Zootecnia pelo Programa de Pós-Graduação em Zootecnia, Universidade Federal de Campina Grande, Patos, Paraíba, Brasil. \\ ${ }^{3}$ Aluno de Mestrado do Programa de Pós-graduação em Medicina Veterinária, Universidade Federal de Campina Grande, Unidade \\ Acadêmica de Medicina Veterinária, Patos, Paraíba, Brasil \\ ${ }^{4}$ Médico veterinário formado pela Universidade Federal de Campina Grande, Unidade Acadêmica de Medicina Veterinária, Patos, Paraíba, Brasil. \\ ${ }^{5}$ Aluna de Graduação em Medicina Veterinária, Universidade Federal de Campina Grande, Unidade Acadêmica de Medicina Veterinária, Patos, Paraiba, Brasil. \\ ${ }^{6}$ Professor do Programa de Pós-graduação em Medicina Veterinária, Universidade Federal de Campina Grande, Patos, Paraíba, Brasil. \\ ${ }^{7}$ Professor do Departamento de Morfologia da Universidade Federal do Rio Grande do Norte, Natal, Brasil. Email: mdanayres@gmail.com \\ *Autor para correspondência, E-mail: jessykacarvalhovet@gmail.com*
}

RESUMO. A doença do disco intervertebral (DDIV) é a causa mais comum de compressão medular em cães, resultando em problemas neurológicos, podendo ser classificada em dois tipos, Hansen tipo I (extrusão de disco) e Hansen tipo II (protrusão de disco), que pressionam os nervos da medula ocasionando quadro de dor, ataxia, paralisia e paraplegia. $\mathrm{O}$ tratamento à se indicar deve ser baseado no grau da lesão, podendo ser o tratamento clínico associado à fisioterapia, para casos menos graves, visto que é relatado sucessos na recuperação do quadro, na literatura. O objetivo desse trabalho foi relatar a eficiência da tomografia computadorizada como ferramenta complementar para diagnostico conclusivo da DDIV e o sucesso do tratamento clínico e fisioterápico para esta doença. O animal em questão era um poodle com 11 anos de idade que apresentava quadro de ataxia e falta de coordenação motora e que foi diagnosticado com uma extrusão de disco intervertebral entre as vertebras T12 e T13 pela tomografia computadorizada. O tratamento eleito foi à base de anti-inflamatórios e fisioterapia com enfoque o fortalecimento da musculatura epaxial e hipaxial. Transcorrido 45 dias do início do tratamento, foi observado uma melhora significativa do animal, entretanto, uma futura intervenção cirúrgica não foi descartada.

Palavras chave: ataxia, compressão medular, fisioterapia, Hansen.

\section{Diagnosis by computed tomography of the intervertebral disc extrusion in a geriatric patient: Case report}

ABSTRACT. Intervertebral disc disease (IVDD) is the most common cause of spinal cord
compression in dogs, resulting in neurological problems, which can be classified into two
types: Hansen type I (disc extrusion) and Hansen type II (disc protrusion), pressing the
nerves of the marrow causing pain, ataxia, paralysis and paraplegia. The indicated
treatment should be based on the degree of injury, and may be the clinical treatment
associated with physical therapy, for less severe cases, based on the successes in the
recovery of the condition, reported in the literature. The objective of this study was to report
the efficiency of computed tomography as a complementary tool for conclusive diagnosis
of DDIV and the success of clinical and physiotherapeutic treatment for this disease. The
animal in question was an 11-year-old poodle that presented ataxia and motor 
incoordination and was diagnosed with an intervertebral disc extrusion between the T12 and $\mathrm{T} 13$ vertebrae by means of computed tomography. The treatment chosen was based on anti-inflammatories and physiotherapy focusing on the strengthening of the epaxial and hipaxial musculature. After 45 days of initiation of treatment, a significant improvement of the animal was observed, however, a future surgical intervention was not ruled out.

Keywords: ataxia, Hansen, physiotherapy, spinal cord compression

\title{
Diagnóstico por tomografía computarizada de la extrusión de disco intervertebral en paciente geriatra: Reporte de un caso
}

\begin{abstract}
RESUMEN. La enfermedad del disco intervertebral (EDIV) es la causa más común de compresión medular en perros, resultando en problemas neurológicos, pudiendo ser clasificada en dos tipos, Hansen tipo I (extrusión de disco) y Hansen tipo II (protrusión de disco), que presionan los nervios de la médula ocasionando cuadro de dolor, ataxia, parálisis y paraplejia. El tratamiento a indicarse debe basarse en el grado de la lesión, pudiendo ser el tratamiento clínico asociado a la fisioterapia, para casos menos graves, ya que en la literatura se reportan recuperaciones exitosas de este tipo de cuadro. El objetivo de este trabajo fue relatar la eficiencia de la tomografía computarizada como herramienta complementaria para diagnóstico conclusivo de la EDIV y el éxito del tratamiento clínico y fisioterápico para esta enfermedad. El animal en cuestión era un Poodle con 11 años de edad que presentaba cuadro de ataxia e incoordinación motora y que fue diagnosticado con una extrusión de disco intervertebral entre las vertebras T12 y T13 a través de tomografía computarizada. El tratamiento elegido fue a base de antiinflamatorios y fisioterapia con enfoque al fortalecimiento de la musculatura epaxial e hipaxial. Transcurrido 45 días del inicio del tratamiento, se observó una mejora significativa del animal, sin embargo, una futura intervención quirúrgica no fue descartada.
\end{abstract}

Palabras clave: ataxia, compresión medular, fisioterapia, Hansen

\section{Introdução}

A doença do disco intervertebral (DDIV) é uma condição facilmente encontrada em cães jovens condrodistróficos ou de meia idade, além dos de grande porte senis como Retrievers, Labrador e Rottweiler, afetando raramente gatos segundo Whindham (2007). Facin et al. (2015) aponta que a doença envolve degeneração do disco e consequente extrusão (Hansen tipo I) ou protrusão (Hansen tipo II) do material discal em direção ao interior do canal vertebral, promovendo compressão medular de intensidades variadas, levando à síndrome neurológica com maior ocorrência em cães.

A DDIV gera deficiências na qualidade e integridade biomecânica do mesmo ocasionando a falha estrutural da unidade funcional da coluna vertebral, podendo causar também a compressão da medula espinhal (Bergknut et al., 2013). A lesão à medula espinhal pode ser aguda ou crônica iniciando uma sequência de eventos vasculares, bioquímicos e inflamatórios. A lesão aguda pode resultar de extrusão de disco intervertebral, êmbolo fibrocartilaginoso ou traumatismo vertebral, a lesão medular crônica pode vir acompanhada de compressão progressiva da medula espinhal e provir de protrusão de disco, neoplasias e instabilidade vertebral (Severo et al., 2011). A manifestação clínica da DDIV ocorre devido a uma combinação do efeito compressivo do material de disco e da lesão de impacto na medula espinhal, decorrente principalmente da extrusão do disco (Sharp \& Wheeler, 2005).

Arias et al. (2007) relatam que dependendo da localização e intensidade da DDIV, há possibilidade de tratamento com analgésicos e anti-inflamatórios, não descartando a chance de recidiva e progressão da doença. Segundo Sharp \& Wheeler (2005) indica-se o tratamento cirúrgico para pacientes com recidiva do quadro ou progressão dos sinais, paraparesia não ambulatória, paraplegia com ou sem percepção de dor profunda.

O presente estudo objetivou relatar a eficiência da tomografia computadorizada como ferramenta complementar para diagnostico conclusivo da DDIV e o sucesso do tratamento clínico e fisioterápico para esta doença. 


\section{Relato de caso}

Um cão macho da raça poodle, com 11 anos de idade e $6 \mathrm{~kg}$ de peso vivo foi atendido em uma clínica particular na cidade de João Pessoa/PB, apresentando leve ataxia e falta de coordenação motora. O proprietário relata que, não houve nenhum trauma anteriormente ou episódio semelhante. $\mathrm{O}$ animal apresentava apetite normal; porém com certa falta de coordenação motora durante o hábito alimentar. Realizou-se um exame de propriocepção ao paciente onde o mesmo não apresentou nenhum déficit proprioceptivo, em seguida, foram realizadas a análise da bioquímica e hematologia sérica, onde ambos estavam dentro dos parâmetros fisiológicos para a espécie, descartando qualquer possibilidade de desarranjos sistêmicos. Levantou-se a hipótese de um acometimento mecânico de caráter ortopédico, em seguida foi realizada uma avaliação tomográfica completa de todo o esqueleto axial do paciente, onde observou-se a ausência de sinais de fratura ou luxação na região avaliada, sem sinais de lesão traumática, porém com a presença de material hiperatenuante localizado no interior do canal vertebral ao nível do espaço intervertebral entre as vértebras torácicas T12 e T13, compatível com extrusão de disco intervertebral (Figura 1), estenose do forâmen intervertebral esquerdo entre as vértebras T12-T13, com dimensão aproximada de $0,8 \mathrm{~mm}$, enquanto o forâmen intervertebral direito apresentou-se com 2,1 mm (Figura 2). Não foram observados sinais de compressão medular evidente ou sinais associados a edema ou hemorragia medular nem de proliferações osteofíticas ou esclerose de placas terminais. Não se evidenciou sinais de neoplasia vertebral. O grau de atenuação radiográfica do osso trabecular das vértebras avaliadas apresentou-se normal, sem sinais de osteopenia ou osteoporose.

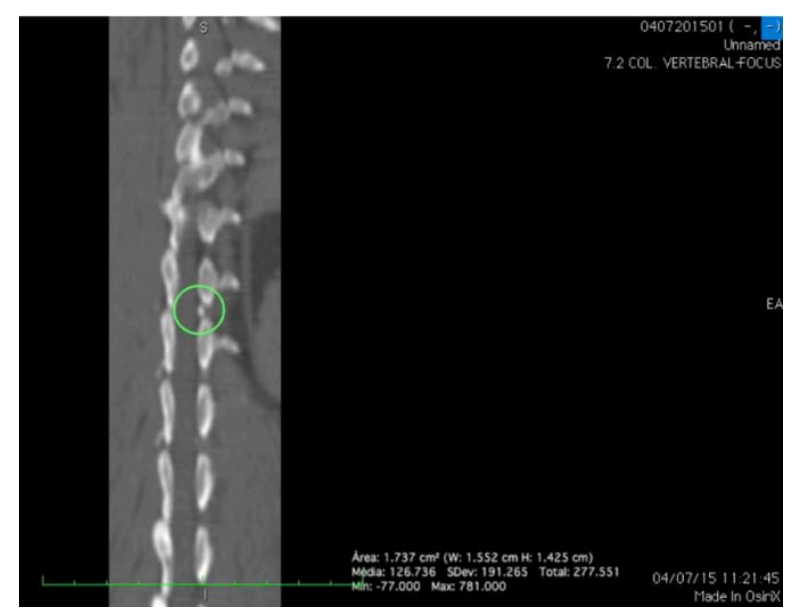

Figura 1. Extrusão de disco intervertebral entre T12 e T13
Após o diagnóstico realizado, o animal foi encaminhado para um tratamento à base de antiinflamatórios e fisioterapia com enfoque $\mathrm{o}$ fortalecimento da musculatura epaxial e hipaxial, com um período de 45 dias observou-se uma melhora significativa na marcha e postura do mesmo, deambulando normalmente e sem dificuldades no hábito alimentar. Apesar da relutância do proprietário em relação ao procedimento cirúrgico, não se descartou a possibilidade de uma intervenção corretiva.

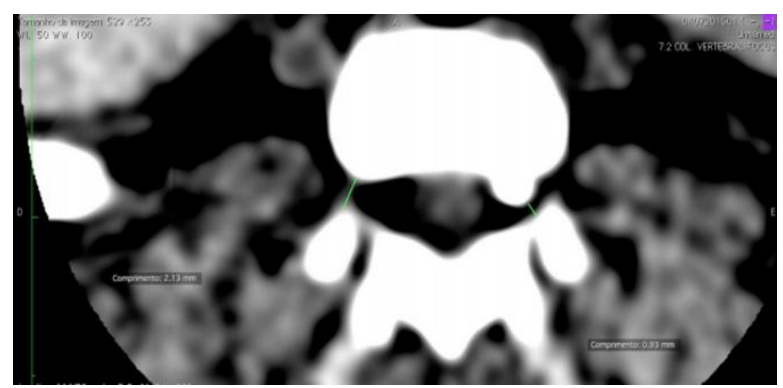

Figura 2. Estenose do forâmen intervertebral esquerdo

\section{Discussão}

As apresentações clínicas das doenças de disco intervertebral variam conforme o grau da lesão, o local de extrusão ou protrusão do material e o nível de inflamação, assim definindo os quadros e intensidade das dores, ataxias e paresia. No presente caso, o animal apresentou ataxia leve, mas sem sinal clínico de dor aguda.

Após a determinação da localização neuroanatômica da lesão medular, torna-se imprescindível realizar exames complementares de diagnóstico para se estabelecer um diagnóstico definitivo (Nelson \& Couto, 2015). Para Fossum (2014), a tomografia computadorizada e a ressonância magnética são capazes de detectar mínimas alterações da medula espinhal que uma radiografia simples ou contrastada não podem revelar. No presente caso, além do histórico e do exame clínico, utilizou-se a tomografia computadorizada, para observar algum possível comprometimento medular e fazer os diagnósticos diferenciais para a demonstração clínica do animal.

A maioria das doenças da coluna vertebral em cães e gatos afetam os segmentos compreendidos entre as vértebras T3 e L3. A DDIV (extrusão ou protrusão) é comumente encontrada neste segmento vertebral, causando compressão da medula espinhal e sinais clínicos que variam desde dor aparente à mielopatia transversa completa. Outras doenças comuns são mielopatia 
degenerativa, trauma espinhal, neoplasia e mielopatia embólica fibrocartilaginosa (Ettinger \& Feldman, 2004, Costa \& Moore, 2010).

Para classificar as diferentes formas da DDIV, precisam-se analisar os dois componentes do disco intervertebral. A primeira porção, o anel fibroso, engloba a parte externa do disco como uma banda fibrosa sendo responsável por conectar as vértebras e permitir a flexibilidade da coluna ao movimento. A segunda parte do disco, o núcleo pulposo, é uma massa gelatinosa que compõe o interior do disco, seu alto teor de água proporciona propriedades elásticas que também conferem flexibilidade e absorção de impactos à coluna (Whindham, 2007).

Arias et al. (2007) em estudo com 45 cães, de diferentes raças, que apresentaram a doença do disco intervertebral tipo I, obtiveram a seguinte prevalência de raças: Dachshund (22/45), seguida pelos cães sem raça definida (7/45), Cocker spaniel (6/45), Lhasa Apso (3/45), Poodle (4/45), Beagle (1/45), Terrier brasileiro (1/45) e Pequinês (1/45). Dependendo da raça, as propriedades do núcleo pulposo desaparecem quando o animal se torna mais velho, o núcleo torna-se desidratado, e a água é gradualmente substituída por cartilagem hialina, colágeno ou minerais (Whindham, 2007). Costa (2001) reportou que os sinais clínicos da DDIV toracolombar geralmente são agudos e progressivos, iniciando com ataxia, progredindo para paraparesia, paraplegia e por fim, paraplegia com ausência de dor profunda.

Em muitos casos, principalmente em animais condrodistróficos, é observada uma evolução superaguda, no qual o proprietário geralmente relata que o cão estava sem qualquer alteração aparente e subitamente apresenta-se paraplégico. Esta manifestação é característica da extrusão (Hansen I) de disco intervertebral toracolombar (Costa, 2001), tal qual pode ser visto na figura 1 onde há extrusão do disco, sendo compatível também com a evolução do quadro do caso, embora não tenha havido a paraplegia, provavelmete devido a menor gravidade da extrusão.

Segundo (Slatter, 2007) o tratamento clínico é indicado para os casos em que apresentam quadros brandos e para os casos sem histórico de recidiva. Inicia-se pela restrição de atividades físicas (confinamento) seguidas por terapia física, uso de medicações anti-inflamatórias ou miorrelaxantes para controlar a dor e hiperestesia. Alguns cuidados auxiliares, sendo fundamental manter o animal em local pequeno, facilitando assim a recuperação do sítio de lesão, diminuindo a inflamação e contribuindo para estabilização do disco por fibrose, estando o tratamento utilizado para o presente caso, de acordo com o reportado pela literatura.

Segundo Ramalho et al. (2015) a fisioterapia deve ser realizada progressivamente, melhorando os déficits neurológicos, prevenindo o desenvolvimento de atrofia muscular e auxiliando no restabelecimento da função motora dos membros paralisados ao mais próximo da normalidade, através de alongamentos, massagens, cinesioterapia, hidroterapia, sempre de acordo com a necessidade de cada paciente. Uma vez realizada em conjunto com o tratamento clínico, a recuperação se torna mais rápida. Tal qual foi observado no relato supracitado, onde em 45 dias houve uma melhora significativa do animal.

As indicações gerais para a intervenção cirúrgica em animais com DDIV são sintomas clínicos não reativos ao tratamento clínico, sintomas clínicos recidivantes ou progressivos, paresia ou paralisia com preservação de sensação dolorosa profunda e paralisia e ausência de dor profunda com duração inferior a 48 horas (Bojrab, 2005, Sharp \& Wheeler, 2005). Todavia, Santos et al. (2011) em um estudo com 20 cães concluiu que metade dos cães com diagnóstico de DDIV sem percepção à dor profunda superior a 48 horas e não submetidos ao tratamento cirúrgico podem apresentar recuperação funcional satisfatória e que é necessário esperar, no mínimo, 30 dias do início dos sinais clínicos para estabelecer um prognóstico quanto ao retorno dos movimentos voluntários.

\section{Conclusão}

A técnica de tomografia computadorizada apresenta confiabilidade para diagnóstico conclusivo na detecção de DDIV quando associada a contraprovas como exames séricos com o intuito de descartar possíveis causas, aumentando a precisão do diagnóstico. Cães portadores de doença do disco intervertebral no segmento toracolombar com pouco agravo podem ter sucesso no tratamento clínico associado à fisioterapia. É de responsabilidade do médico veterinário o diagnóstico baseado em exames e a recomendação do tratamento adequado em consonância com o grau da lesão, objetivando a qualidade de vida do animal. 


\section{Referências Bibliográficas}

Arias, M. V. B., Nishioka, C. M., Garcia, C. O., Reia, A. Z., Júnior, D. B. \& Marcasso, R. A. 2007. Avaliação dos resultados clínicos após cirurgia descompressiva em cães com doença de disco intervertebral Evaluation of clinical results of decompressive surgery in dogs with degenerative disk disease. Arquivo Brasileiro de Medicina Veterinária e Zootecnia, 59, 1445-1450.

Bergknut, N., Smolders, L. A., Grinwis, G. C. M., Hagman, R., Lagerstedt, A.-S., Hazewinkel, H. A. W., Tryfonidou, M. A. \& Meij, B. P. 2013. Intervertebral disc degeneration in the dog. Part 1: Anatomy and physiology of the intervertebral disc and characteristics of intervertebral disc degeneration. The Veterinary Journal, 195, 282-291.

Bojrab, M. J. 2005. Técnicas atuais em cirurgia de pequenos animais. Editora Roca, São Paulo.

Costa, R. C. 2001. Disco intervertebral: bases para o diagnóstico e tratamento da doença. Nosso Clinico, 4, 20.

Costa, R. C. \& Moore, S. A. 2010. Differential diagnosis of spinal diseases. Veterinary Clinics of North America: Small Animal Practice, 40, 755-763.

Ettinger, S. \& Feldman, E. 2004. Tratado de medicina interna veterinária: doenças do cão e do gato. Guanabara Koogan, Rio de Janeiro.

Facin, A. C., Rocha, T. A. S. S. \& Watanabe, B. 2015. Doença de disco intervertebral em cães: 16 casos. Enciclopédia Biosfera - Centro Científico Conhecer., 11, 21.

Fossum, T. W. 2014. Cirurgia de pequenos animais, 4 edn. Elsevier Brasil, São Paulo.
Nelson, R. W. \& Couto, C. G. 2015. Medicina interna de pequenos animais. Elsevier Editora, Amsterdan.

Ramalho, F. P., Formenton, M. R., Isola, J. G. M. P. \& Joaquim, J. F. G. 2015. Tratamento de doença de disco intervertebral em cão com fisioterapia e reabilitação veterinária: relato de caso. Revista de Educação Continuada em Medicina Veterinária e Zootecnia do CRMVSP, 13, 10-17.

Santos, R. P., Mazzanti, A., Beckmann, D. V., Berté, L., Ripplinger, A., Neto, D. P. \& Baumhardt, R. 2011. Recuperação funcional em cães com doença do disco intervertebral toracolombar sem percepção à dor profunda: 37 casos (2002-2010). Pesquisa Veterinária Brasileira, 31, 345-349.

Severo, M. S., E.A., T. \& M.V.B, A. 2011. Fisiopatologia do trauma e da compressão à medula espinhal de cães e gatos. Medicina Veterinária, 1, 78-85.

Sharp, N. J. \& Wheeler, S. J. 2005. Small animal spinal disorders: diagnosis and surgery. Elsevier Mosby, Philadelphia, EUA.

Slatter, D. H. 2007. Manual de cirurgia de pequenos animais. Manole, São Paulo.

Whindham, R. 2007. Intervertebral Disk Disease. Veterinary Technician, 28, 1-10.

Article History:

Received 7 November 2017

Accepted 14 December 2017
Available online 10 February 2018

License information: This is an open-access article distributed under the terms of the Creative Commons Attribution License 4.0, which permits unrestricted use, distribution, and reproduction in any medium, provided the original work is properly cited. 